\title{
Cold storage induces rat liver pyroptosis by activating endoplasmic reticulum stress response through the ATF6-CHOP pathway
}

jiang liu

The Affiliated Hospital of Southwest Medical University

Tiancheng Liang

Luzhou Municipal Hospital of Traditional Chinese Medicine

\section{Yong $\mathrm{Ni}$}

The Hospital of Chongqing Shapingba district people`s

De Luo

The Affiliated Hospital of Southwest Medical University

Bo Li

The Affiliated Hospital of Southwest Medical University

\section{Yu Gan}

The Affiliated Hospital of Southwest Medical University

\section{Cheng Fang}

The Affiliated Hospital of Southwest Medical University

\section{Santao Ou}

The Affiliated Hospital of Southwest Medical University

\section{Song Su ( $\nabla 48115251 @ q q . c o m$ )}

The Affiliated Hospital of Southwest Medical University https://orcid.org/0000-0002-6060-8654

\section{Research}

Keywords: Liver, Cold storage, Pyroptosis, Endoplasmic reticulum stress

Posted Date: February 26th, 2021

DOI: https://doi.org/10.21203/rs.3.rs-184227/v1

License: (c) (i) This work is licensed under a Creative Commons Attribution 4.0 International License. Read Full License 


\section{Abstract}

Aims :Liver injury is a common complication of cold storage (CS), and often constitutes a direct cause for liver transplantation failure. The cellular and molecular mechanisms underlying CS-induced liver injury remain unclear. Recent evidence indicates that pyroptosis plays an important role in multiple pathophysiological processes. Using rat liver tissue and cells as a model, we identified a novel mechanism by which inflammasome-dependent interleukin-1 $\beta$ (IL-1 $\beta)$ activation and hepatocyte pyroptosis mediate CS-induced liver injury.

Methods :To induce CS, liver tissue and cells were subjected to storage at $4^{\circ} \mathrm{C}$ for 12 and $24 \mathrm{~h}$. Inhibition of endoplasmic reticulum (ER) stress was achieved by RNA silencing. Measurements of caspase-1, caspase-11, and IL-1 $\beta$ were performed.

Results: Pyroptosis was activated in CS-treated livers, as evidenced by increased levels of caspase-1 and caspase-11 activity, and the elevated expression of IL-1 1 . ER stress response was activated as well. Inhibition of ER stress response prevented CS-induced liver pyroptosis and inflammation.

Conclusion : Our findings suggest that pyroptosis might be playing an important role in the development of liver injury induced by CS. Overactivated ER stress response, followed by activation of the ATF6-CHOP signaling pathway, might be a novel molecular mechanism involved in CS-induced pyroptosis of liver tissue and cells.

\section{Introduction}

Liver transplantation is currently an established treatment for patients suffering from end-stage liver disease. Although significant progress in liver transplantation has been made over the past two decades, cold injury in cold-stored (CS) liver remains a major challenge to be overcome [1,2]. Extensive body of evidence indicates that apoptosis plays an important role in hepatic tissues injury [3-5]. Although a large number of experiments have been performed in an attempt to minimize apoptosis and its effects on transplanted organs, results are still not satisfactory. Whether other tissue- and cell-loss mechanisms are involved in transplanted organ injury requires further in-depth research.

Pyroptosis is a unique type of programmed cell death that is distinct from apoptosis and necrosis $[6,7]$. Pyroptosis depends on the activation of the caspase- 1 cascade and the formation of inflammatory cytokines, including those of the IL-1 family. A recent study reported a link between cell pyroptosis and renal ischemia-reperfusion injury[8]. CS is a very important intermediate stage preceding organ transplantation, and organs often suffer from cold ischemic damage during storage. Whether pyroptosis is involved in liver cells death when stored at low temperatures, in this study we explored the effects of CS on liver tissue and cells. Whatever, pyroptosis and inflammatory cytokines are related to such damage remain unknown,So we firstly elucidated the association between CS and cells' pyroptosis.

\section{Materials And Methods}




\section{Animal care, rat model, and treatments}

All animal procedures were conducted in compliance with protocols approved by the local government ethical authorities. Eight to 12 weeks old male Sprague Dawley (SD) rats, weighing 250-300 g, were purchased from the Experimental Animal Center of Southwest Medical University (Luzhou, Sichuan, China). The rats were treated humanely, and all experiments were performed under conditions designed to minimize suffering. SD rats were randomly divided into three groups. Un-treated blank control (Group 1, $n=6$ ), liver tissue CS for $12 \mathrm{~h}$ (Group 2, $n=6$ ), liver tissue CS for $24 \mathrm{~h}$ (Group 3, $\mathrm{n}=6$ ). Briefly, rats were euthanized by intramuscular injection of sodium pentobarbital (30 $\mathrm{mg} \mathrm{kg}^{-1}$ of body weight). After opening the abdominal cavity, the livers were procured and flushed with $30 \mathrm{~mL}$ of University of Wisconsin (UW) solution. Livers of treatment groups 2 and 3 were then stored in the UW solution at $4^{\circ} \mathrm{C}$ for 12 or 24 h, respectively.

\section{Cell culture and intervention}

Buffalo rat liver (BRL) cells from normal rat liver were obtained from the cell bank of the Chinese Academy of Sciences (Shanghai, China), and cultured in Dulbecco's modified Eagle medium (DMEM) (Gibco, Invitrogen, Carlsbad, CA, USA), supplemented with 10\% fetal bovine serum (Gibco, Invitrogen), 100 $\mathrm{U} \mathrm{mL}^{-1}$ penicillin, and $100 \mu \mathrm{g} \mathrm{mL}^{-1}$ streptomycin. For cold treatment, the cells were incubated at $4^{\circ} \mathrm{C}$ in an atmosphere of $5 \% \mathrm{CO}_{2}$ in air. The BRL cells were also treated with ATF6-specific small interfering RNA (siRNA), using Lipofectamine 2000 (Invitrogen, Grand Island, NY) in the culture medium. Untreated cells acted as control. The cells were cultured at $44^{\circ} \mathrm{C}$ and an atmosphere of $5 \% \mathrm{CO}_{2}$ in air for $12 \mathrm{~h}$ and $24 \mathrm{~h}$. The primers' sequences were as follow:

Forward: 5-TCCTCGGTCAGTGGACTCTTA-3,

Reverse: 5-CTTGGGCTGAATTGAAGGTTTTG-3.

\section{Histopathology and immunohistochemistry}

Liver samples were fixed in $4 \%$ neutral formaldehyde, dehydrated in a gradient series of ethyl alcohol, dealcoholized in xylene, and then embedded in paraffin. Liver tissue samples were sectioned at $4 \mu \mathrm{m}$, mounted on slides and stained with hematoxylin and eosin (HE). Severity of liver damage was estimated by evaluating five randomly-selected low-magnification (x100) fields in each HE-stained section. Liver oxidative stress was detected using gp $91^{\text {phox }}$ immunohistochemical analysis. Briefly, after antigen retrieval, the sections were incubated with gp9 ${ }^{\text {phox }}$ antibody (Abcam, Cambridge, USA) for $1 \mathrm{~h}$ at room temperature. The slides were then washed with $0.5 \% \mathrm{BSA}$ and incubated with a secondary antibody (Abcam, Cambridge, USA) for 60 min. Subsequently, antigen-antibody complexes were detected using diaminobenzidine tetrahydrochloride (DAB; Sigma-Aldrich, St. Louis, MO, USA) as a chromogen. Nuclei were counterstained with hematoxylin.

\section{Western blot analysis}


Cells were harvested and homogenized with cell lysis buffer (Beyotime, China). Total protein in the lysates was quantified using BCA Protein Assay Kit (Beyotime, China). Equal quantities of protein samples were separated by denaturation with $10 \%$ SDS/PAGE and then transferred onto polyvinylidene difluoride (PVDF) membranes. Membranes were incubated in a 5\% skim milk TBST blocking solution at room temperature (RT) for $1 \mathrm{~h}$. The membranes were then incubated with primary antibody, followed by horseradish peroxidase-conjugated secondary antibody. Finally, protein bands were visualized using an enhanced chemiluminescence (ECL) western blotting detection system (GE Healthcare, Amersham, UK). Antibodies used in this study were: cleaved caspase-12, GRP78, ATF6, CHOP, caspase1, caspase-11, IL-1 3 , GAPDH, and $\beta$-actin. All antibodies were purchased from Abcam (Cambridge, MA, USA) or Cell Signaling Technology (Beverly, MA, USA).

\section{Statistical analysis}

Data are reported as mean \pm standard deviation (SD). Comparisons between groups were performed using the Student's t-test. Multiple groups were compared using ANOVA. The overall survival was analyzed by Pearson's correlation and Kaplan-Meier survival analysis. Differences were considered significant when $P<0.05$.

\section{Results}

In this study, we found that the level of the pyroptosis-related proteins, including caspase-1, caspase-11, and IL-1 $\beta$, significantly increased after $12 \mathrm{~h}$ of liver tissue cold-storage and peaked after CS of $24 \mathrm{~h}$. Similarly, CS induced pyroptosis in BRL cells, as was evident from the increase in lactate dehydrogenase release and the pathological changes in the samples. In addition, clear upregulation of the endoplasmic reticulum (ER) stress biomarkers, activator of transcription factor 6 (ATF6) and C/EBP homologous protein (CHOP), preceded pyroptosis in cells treated with CS. Silencing of ATF6 with siRNA significantly decreased CS-induced pyroptosis of BRL cells, as evidenced by reduced caspase- 1 and caspase- 11 activity, and IL-1 $\beta$ generation. We therefore conclude that pyroptosis, resulting from ER stress and ATF6CHOP activation, is an important process, contributing to the damage seen in CS-treated BRL cells.

\section{Cold storage induces injury in rats' liver samples}

To determine whether there is pyroptosis, and if so - what is its potential function in liver cold injury, SD Rats' livers were used as a CS model. As shown in Figure 1, after liver samples were maintained in UW solution at $4^{\circ} \mathrm{C}$ for $12 \mathrm{~h}$ or $24 \mathrm{~h}$, they showed CS-related injury. HE staining was used to evaluate embedded tissue slices. As shown in Fig $1 \mathrm{~A}$, focal loss of cells' integrity, karyopyknosis, and dilated sinusoids are evident in samples from both cold-storage groups, notably more after $24 \mathrm{~h}$ of cold storage. The Suzuki scoring method was used to score liver pathology in each group (Fig 1B). Measurement of lactate dehydrogenase (LDH)| served as a marker for hepatocellular injury. As shown in Figure 1C, perfusate enzymes activity was markedly elevated in CS-treated livers when compared with sham-treated control livers. These pathophysiological changes indicate that the cold treatment was successful in inducing hepatic injury, justifying its use as a model in our study. 


\section{Cold storage induces pyroptosis and inflammation in liver tissues}

Pyroptosis was defined by the presence of active caspase- 1 or caspase- 11 . To determine whether activation of pyroptosis in hepatocytes is the result of CS, expression of pyroptosis-related proteins, namely caspase- 1 , caspase- 11 , and IL-1 $\beta$ was assessed. Western blot and

immunohistochemistry analysis revealed that the levels of all three proteins were markedly elevated at 12 $\mathrm{h}$, and peaked at $24 \mathrm{~h}$ of CS (Fig 2). These results suggest that CS induces liver tissues pyroptosis and

inflammation.

\section{Cold storage can trigger ER stress}

It has been reported that damages to the ER and mitochondria are implicated in cold stress-induced apoptosis [9-10]. Furthermore, previous studies reported that ER stress protein ATF6 is activated and mediates apoptosis in liver cells during CS. In this experiment, expression of ER stress-related proteins was studied. Western blot results (Fig 3 ) show that the expression level of the ER stress-related proteins, GRP78, ATF6, and CHOP, has significantly increased following CS, as compared to the sham-treated control. Moreover, levels of these proteins showed a time-dependent expression. They were markedly elevated after $12 \mathrm{~h}$ of CS and peaked after $24 \mathrm{~h}$ of CS.

\section{Cold storage induces pyroptosis and ER stress in BRL cells}

It is imperative to also investigate the effect of CS of BRL cells on the expression of pyroptosis-related proteins, including those related to ER stress. As shown in Figure 4, GRP78, ATF6, CHOP, caspase-1, caspase-11, and IL-1 $\beta$ were all expressed at low levels in untreated BRL cells. Following $12 \mathrm{~h}$ of CS, expression of these proteins was significantly upregulated. Levels have further increased when the cells were treated with $24 \mathrm{~h}$ of CS. These results suggest that, just like with liver tissue, CS of BRL cells induces pyroptosis and ER stress.

\section{The ATF6-CHOP pathway is involved in cold storage-induced pyroptosis of BRL cells}

To confirm the association between ATF6-CHOP and pyroptosis following CS of BRL cells, further experiments were carried out. Based on the results reported above, injury to hepatic cells and tissues was most severe following $24 \mathrm{~h}$ of CS. We therefore selected $24 \mathrm{~h}$ CS treatment to evaluate the significance of ATF6-mediated pyroptosis. After AFT6 silencing in BRL cells that were exposed to $24 \mathrm{~h}$ of CS, levels of ER stress-related proteins were investigated. As shown in Figure 5A, compared with the control group, no obvious changes took place in the levels of CHOP in the ATF6 siRNA-treated group.

Changes in the expression level of pyroptosis-related proteins after $24 \mathrm{~h}$ of CS were also evaluated. As shown in Figure 5 A, levels of caspase-1, caspase-11, and IL-1 $\beta$ remained similar to those in the control group when the BRL cells were treated with ATF6 siRNA. The ATF6 siRNA-treated BRL cells markedly decreased the activation of inflammatory caspases as well as the levels of IL-1 $\beta$. These results indicate that exposure of BRL cells to $24 \mathrm{~h}$ of CS results in activation and overexpression of GRP78, ATF6, and 
CHOP. These, in turn, activates hepatocyte pyroptosis. This conclusion is supported by the fact that ATF6 miRNA blocks this chain of events. Taken together, these results indicate that the ATF6-CHOP pathway is involved in CS-induced pyroptosis of BRL cells.

\section{Discussion}

At present, under normal conditions, livers are stored at $2-8^{\circ} \mathrm{C}$ as whole organs, perfused in UW media or histidine-tryptophan-ketoglutarate solution (HTK) [11-12]. These solutions facilitate extending liver preservation time from several hours to a maximum of a few days. However, it has been shown that CS causes vasoconstriction, tubular and endothelial injury, and cell death [13]. These may lead to liver dysfunction or delayed graft function upon transplantation. Although the issue was discussed in many studies, the cellular and molecular mechanisms leading to hepatic cold-induced injury remain poorly characterized. Understanding the underlying mechanisms and devising ways to attenuate them could have a tremendous impact on the number of transplants performed, as well as on the outcome of liver transplantation procedures.

Pyroptosis is a programmed cell death characterized by rapid plasma membrane rupture and release of pro-inflammatory intracellular contents. The pathway is morphologically and mechanistically distinct from other forms of cell death. A defining feature of pyroptosis is its dependence on caspase-1 [14]. Caspase- 1 was first recognized as a protease that cleaves the inactive precursors of IL-1 $\beta$ and IL-18 into mature inflammatory cytokines, and it was initially called IL-1 $\beta$ converting enzyme [15]. Activation of caspase- 1 induces pore formation on the cell membrane and contributes to the generation and release of abundant inflammatory factors, thus contributing to pyroptosis [16-17]. Caspase-11, which is the upstream regulator of caspase-1, mediates its activity by direct cleavage of pro-caspase-1 [18]. Recent studies[19-20] have shown that caspase-11 can activate caspase-1 under the coordination of NLRP3 inflammasomes and can also induce caspase-1-independent pyroptosis. These findings denote that caspase-11 mediates pyroptosis in a caspase-1-dependent or -independent manner; and, stemming from this, the potentially important role both caspase-1 and caspase-11 play in pyroptosis [21]. Our in vitro study has shown that pyroptosis is characterized by an increase in the expression of caspase- 1 and caspase-11, and generation of IL-1 $\beta$, that already appeared after 6 hland peaked after $24 \mathrm{~h}$ of CS. The altered levels of caspase-1, caspase-11, and IL-1 $\beta$ in liver tissues positively correlated with CS duration. Our in vitro study revealed that CS upregulated caspase-1 and caspase-11 in normal liver cells. This was accompanied by increased pore formation, LDH release, and IL-1 $\beta$ generation. These results suggest that when cells undergo pyroptosis, they lose membrane integrity, which leads to the release of cellular contents, including LDH and the inflammatory cytokine IL-1 $\beta$.

Endoplasmic reticulum (ER) stress is an initiator of cell death and inflammatory mechanisms. A number of studies have shown that by activating ER stress, ischemia-reperfusion injury (IRI) can induce several cell death mechanisms, including autophagy, apoptosis, necroptosis, and mitochondria-mediated programmed necrosis [22-24]. A few studies on pancreatic $\beta$-cells [25-26] have demonstrated that ER stress can activate the NOD-like receptor (NLR) family, pyrin domain-containing 3 (NLRP3) 
inflammasome via protein kinase RNA-like ER kinase, and inositol-requiring enzyme 1 signaling pathways, thus triggering caspase- 1 activity, and leading to cells death and IL-1 $\beta$ release. Yang and colleagues [8] were first to demonstrate that the expression of GRP78 and CHOP was significantly elevated in renal IRI in mice. CHOP (C/EBP homologous protein) can induce the expression of casepase11 and activate its effector, activator casepase-11. Casepase-11 overexpression promotes activation of the cytokines IL-1 $\beta$ and IL-18, and induces pyroptosis. Inhibiting the expression of CHOP would weaken the activity of caspase-11. Pretreatment with the ER stress inducer ionomycin can alleviate pyroptosis induced by IRI, suggesting that CHOP-caspase- 11 is an important signaling pathway related to pyroptosis during renal ischemia-reperfusion. It is thus clear that over activated ER stress might be an important cause of pyroptosis in certain cell types.

The upregulation of GRP78 is a hallmark for the initiation of ER stress. Once initiated, ATF6 is an important signaling molecule associated with the process. Elevated expression of $\mathrm{CHOP}$, a downstream molecule, indicates the activation of the ER-stress-mediated cell death signaling pathway. Hence, upregulation of GRP78,ATF6 and CHOP indicates over activated ER stress [27]. Our previous study [28] showed that ER stress is in an active state during CS of the liver, with GRP78, ATF6, and CHOP highly expressed. However, knowledge was lacking with respect to the involvement of pyroptosis in liver cell death following CS and its relationship with the release of inflammatory cytokines. Our objective in this study was thus to investigated the pyroptosis-related alterations that occur in tissues and cells of the liver when exposed to CS. Our results show that upregulation of GRP78, ATF6, and CHOP may precede pyroptosis, as indicated by increased pore formation, LDH release, elevated expression of caspase-1 and caspase- 11 , and increased production of IL-1 $\beta$ in liver tissue and cells treated with CS. Furthermore, our results show that pyroptosis-related proteins expression is positively correlated to the duration of CS treatment. Understanding the relationship between the duration of CS and liver damage would direct us to the realization that shortening the time of CS would help reduce pyroptosis. Our in vitro study demonstrated that silencing ATF6 significantly suppressed CS-induced BRL cell pyroptosis, supporting the notion that pyroptosis might be a downstream effector of overactivated ER stress.

Pyroptosis is a unique programmed cell death, distinct from apoptosis and necrosis. Here we showed that overactivated ER stress followed by activation of the ATF6-CHOP signaling pathway might be a novel molecular mechanism involved in CS-induced pyroptosis of hepatic tissue and BRL cells. Moreover, the severity of pyroptosis is closely related to hepatic tissue injury.

\section{Declarations}

\section{Compliance with ethical standards}

Conflict of interest The authors declares that they have no conflict of interest.

\section{Acknowledgments}

This work was supported by Luzhou Science and Technology Bureau innovation Seedling program (no.2017-R69) 


\section{References}

1. Ploeg RJ, D'alessandro AM, Knechtle SJ, et al.Risk factors for primary dysfunction after liver transplantation-a multivariate analysis[J].Transplantation ,1993,55: 807-

2. 813 .

3. 2. Varotti G, Grazi GL, Vetrone G, et al.Causes of early acute graft failure after livertransplantation: analysis of a 17-year single-centre experience[J]. Clin Transplant,2005,19:492-500.

4. 3. Mosbah IB, Zaouali MA, Martel C, et al. IGL-1 solution reduces endoplasmic reticulum stress and apoptosis in rat liver transplantation[J]. Cell Death Dis,2012, 3:e279.

5. 4. Baskin-Bey E, Canbay A, Bronk S,et al. Cathepsin B inactivation attenuates hepatocyte apoptosis and liver damage in steatotic livers after cold ischemia-warm reperfusion injury[J]. Am J Physiol Gastrointest Liver Physiol,2005, 288:G396-G402.

6. 5. Stolz DB, Ross MA, lkeda A,et al. Sinusoidal endothelial cell repopulation following ischemia/reperfusion injury in rat liver transplantation[J]. Hepatology,2007, 46:1464-1475.

7. 6. Bergsbaken T, Fink SL, Cookson BT. Pyroptosis: host cell death and inflammation[J]. Nat Rev Microbiol ,2009,7: 99-109.

8. 7. Miao EA, Rajan JV, Aderem A. Caspase-1-induced pyroptotic cell death[J]. Immunol Rev ,2011,243: 206-214.

9. 8. Yang JR, Yao FH, Zhang JG, et al. Ischemia-reperfusion induces renal tubule

10. pyroptosis via the CHOP-caspase-11 pathway[J]. Am J PhysiolRenal Physiol, 2014,

11. 306. (1): F75-F84.

12. 9. Salahudeen $A K$, Huang $H$, Joshi $M$, et al.Involvement of the mitochondrial pathway in cold storage and rewarming-associated apoptosis of human renal proximal tubular cells[J]. Am J Transplant ,2003,3:273-280.

13. 10. Zhu X, Zelmer A, Kapfhammer JP ,et al.Cold-inducible RBM3 inhibits PERK phosphorylation through cooperation with NF90 to protect cells from endoplasmic reticulum stress[J]. FASEB J, 2015,30:1-11.

14. 11. Hwang S, Lee SG, Joh JW, et al.Liver transplantation for adult patients with hepatocellular carcinoma in Korea: comparison between cadaveric donor and living donor liver transplantations[J]. Liver Transpl ,2015,11:1265-1272.

15. 12. Mangus RS, Tector AJ, Agarwal A, et al.Comparison of histidine-tryptophan-

16. ketoglutarate solution (HTK) and University of Wisconsin solution (UW) in adult

17. liver transplantation[J]. Liver Transpl ,2006,12: 226-230.

18. 13. Salahudeen AK .Cold ischemic injury of transplanted kidneys: new insights from experimental studies[J]. Am J Physiol Renal Physiol,2004, 287: F181-F187.

19. 14. Bergsbaken T, Fink SL, Cookson BT. Pyroptosis: host cell death and inflammation[J]. Nat Rev Microbio,2009,I 7: 99-109. 
20. 15. Alnemri ES, Livingston DJ, Nicholson DW, et al. Human ICE/CED-3 protease nomenclature[J].Cell,1996, 87: 171.

21. 16. Bergsbaken $\mathrm{T}$, Cookson BT. Macrophage activation redirects yersiniainfected host cell death from apoptosis to caspase-1-dependent pyroptosis[J].PLoS Pathog ,2007,3: e161.

22. 17. Fink SL, Cookson BT. Caspase-1-dependent pore formation during pyroptosis leads to osmotic lysis of infected host macrophages[J]. Cell Microbiol ,2006,8: 1812-1825.

23. 18. Wang $S$, Miura $M$, Jung $Y K$, et al. Murine caspase-11, an ICE-interacting protease, is essential for the activation of ICE[J]. Cell ,1998,92:501-509.

24. 19. Kayagaki $\mathrm{N}$, Warming $\mathrm{S}$, Lamkanfi $\mathrm{M}$, et al. Non-canonical inflammasome activation targets caspase-11[J]. Nature ,2011,479: 117-121.

25. 20. Yeh $\mathrm{CH}$, Hsu SP, Yang CC, et al. Hypoxic preconditioning reinforces HIF-?-

26. dependent HSP70 signaling to reduce ischemic renal failure-induced renal tubular apoptosis and autophagy[J]. Life Sci, 2010,86:115-123.

27. 21. Aachoui Y, Leaf IA, Hagar JA, et al. Caspase-11 protects against bacteria that escape the vacuole[J]. Science ,2013,339: 975-978.

28. 22. Jager R, Bertrand MJ, Gorman AM, et al. The unfolded protein response at the crossroads of cellular life and death during endoplasmic reticulum stress[J]. Biol Cell ,2012,104: 259-270.

29. 23. Lee $H, P a r k M T$, Choi BH, et al. Endoplasmic reticulum stress-induced JNK activation is a critical event leading to mitochondria-mediated cell death caused by ?-lapachone treatment[J]. PLos One,2011, 6: e21533.

30. 24. Su J, Zhou L, Kong X, Yang X, et al. Endoplasmic reticulum is at the crossroads of autophagy, inflammation, and apoptosis signaling pathways and participates in the pathogenesis of diabetes mellitus[J]. J Diabetes Res, 2013: 193461.

31. 25. Menu P, Mayor A, Zhou R, et al. ER stress activates the NLRP3 inflammasome via an UPRindependent pathway[J]. Cell Death Dis,2012, 3: e261.

32. 26. Oslowski CM, Hara T, O'Sullivan-Murphy B, et al. Thioredoxin-interacting protein mediates ER stress-induced ?cell death through initiation of the inflammasome[J]. Cell Metab ,2012,16: 265-273.

33. 27. Han J, Back SH, Hur J, et al. ER-stress-induced transcriptional regulation increases protein synthesis leading to cell death[J]. Nat Cell Biol ,2013,15: 481-490.

34. 28. Su S, Liu J, He K, et al. Overexpression of the long noncoding RNA TUG1 protects against coldinduced injury of mouse livers by inhibiting apoptosis and inflammation[J]. Febs Journal, 2016, 283(7):1261.

\section{Figures}




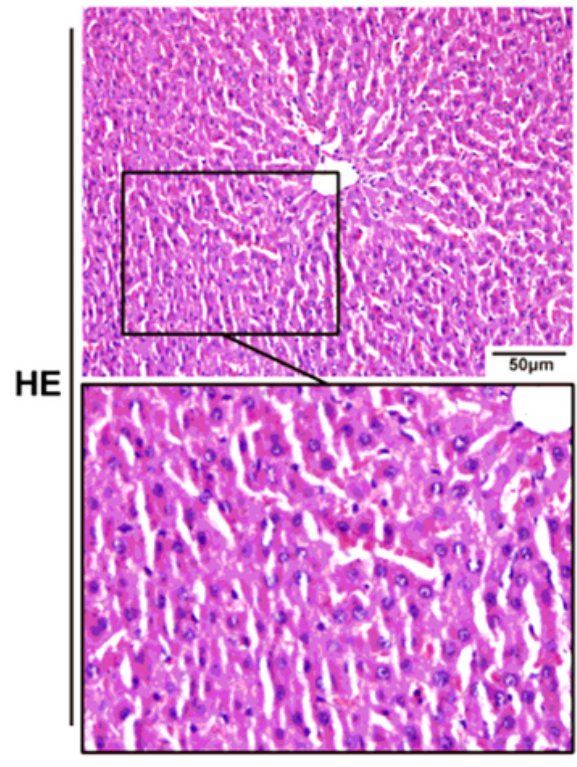

B

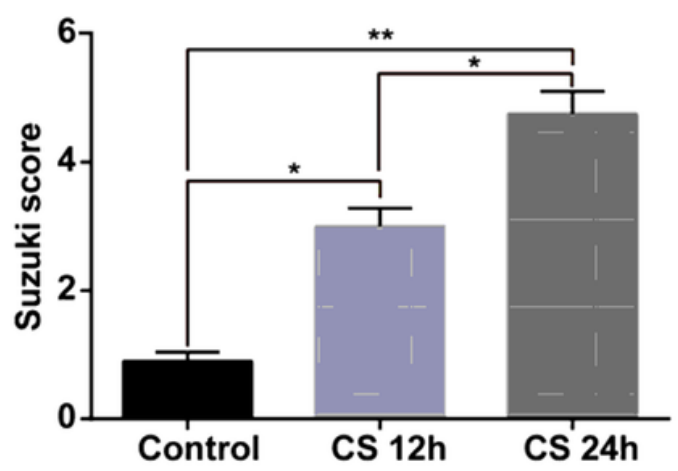

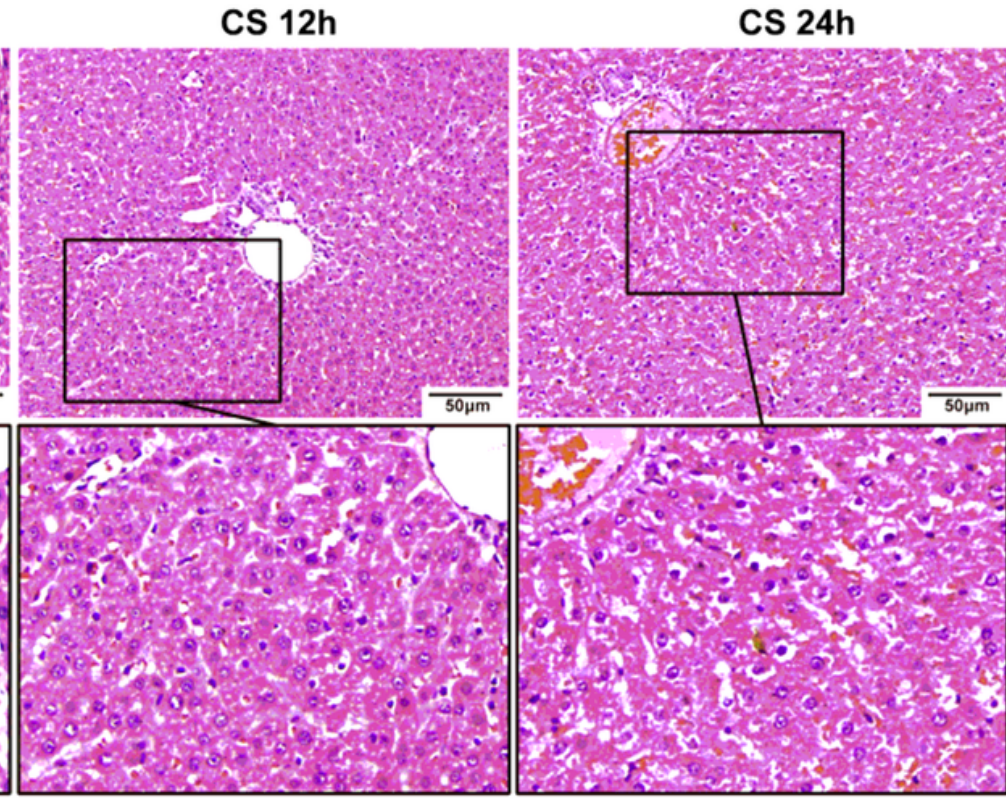

C

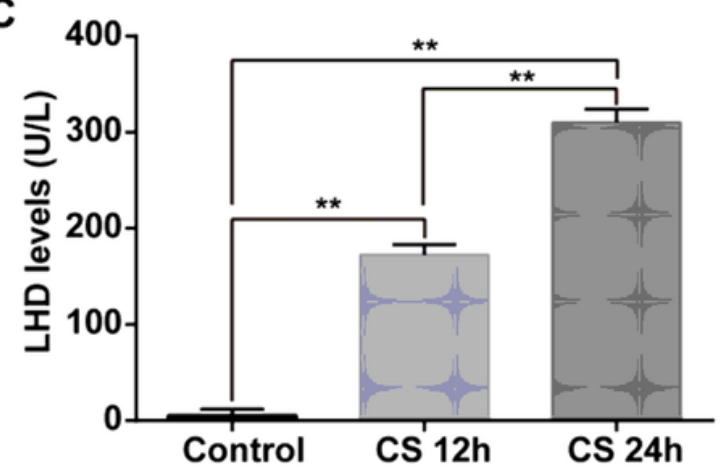

Figure 1

Characteristics of rat liver injury after cold-storage treatment. (A) Micrographs of representative HE stained rats' liver sections after $12 \mathrm{~h}$ and $24 \mathrm{~h}$ of cold storage. (B) Suzuki score of liver tissue in each experimental group. Data are presented as means + SD. Statistical analysis was performed by Student's ttest, $\mathrm{n}=6$ rats per group. (C) Perfusate LDH levels, analyzed as a measure of hepatocellular injury. ${ }^{*} \mathrm{P}<$ $0.05, * \star \mathrm{P}<0.01$. 

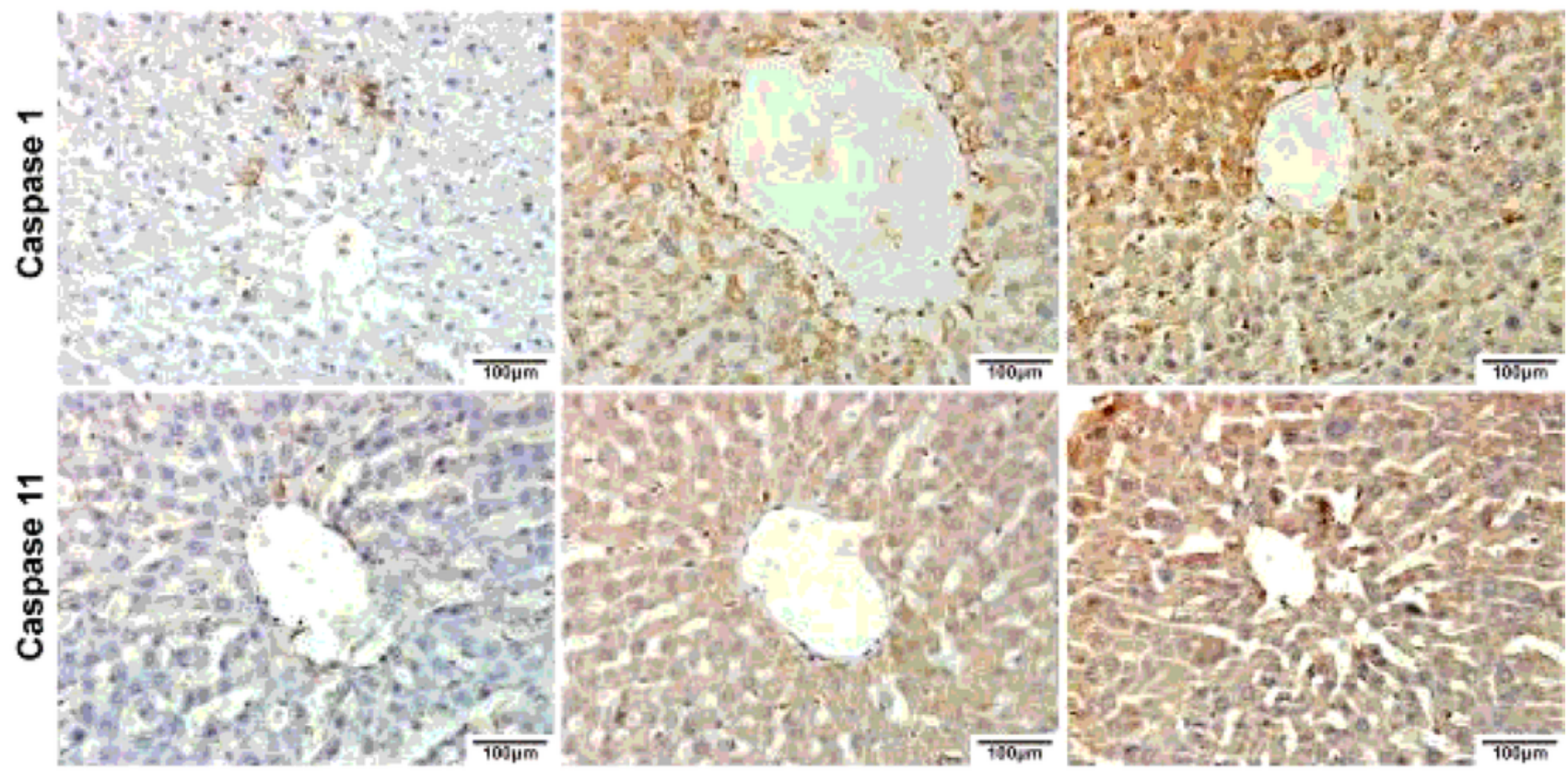

B
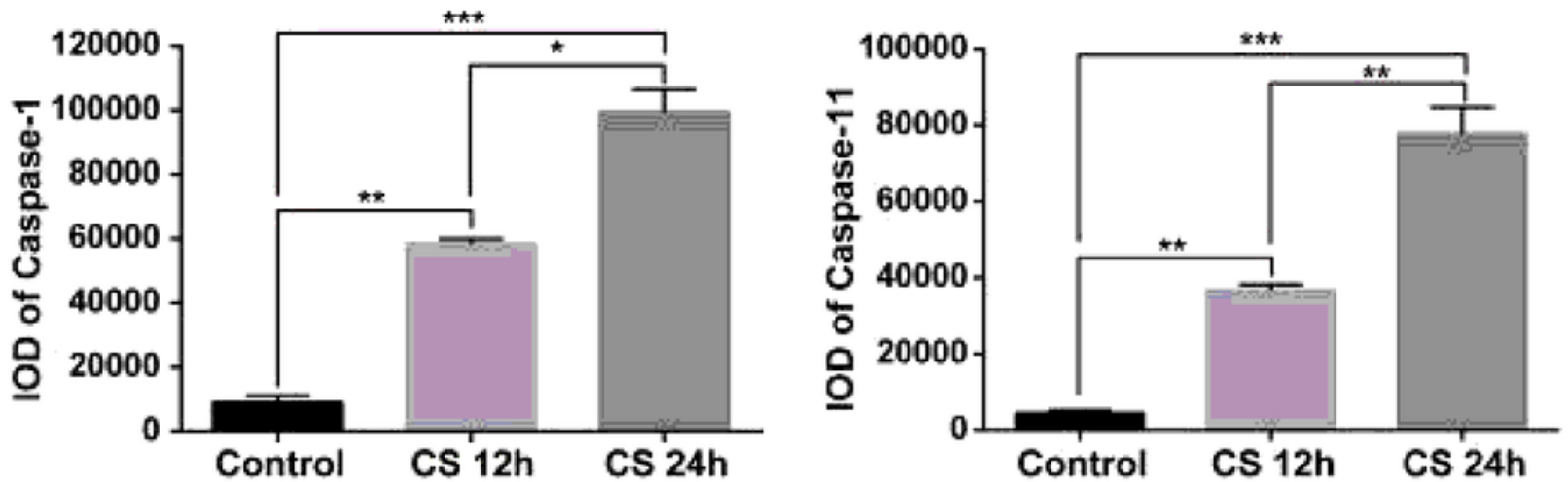

A

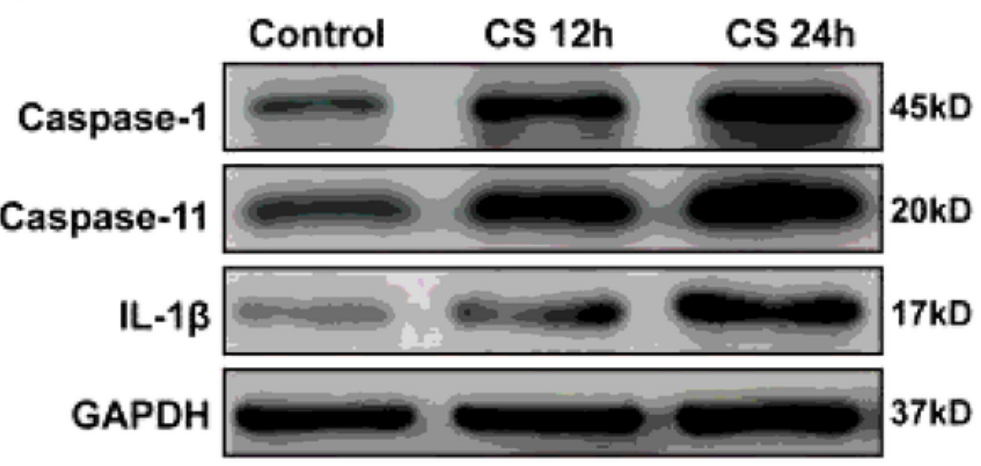

B

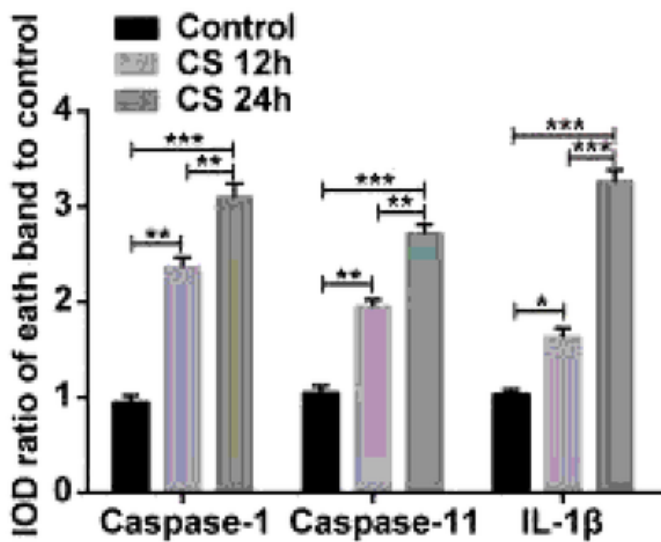

Figure 2

Cold storage altered the levels of pyroptosis-associated proteins. Levels of caspase- 1 and caspase-11 in rat hepatic tissues characterized by immunohistochemistry assays after CS for specific time durations (A). Protein expression level of caspase-1, caspase-11, IL-1 13 , and GAPDH in hepatic tissues examined by Western blot analysis $(\mathrm{B}, \mathrm{C})$. Images are true representation of the samples. Data shown are expressed as mean + SD of individual groups $\left(n=6\right.$ rats/group at each time point). ${ }^{*} P<0.05$, ${ }^{\star *} P<0.01,{ }^{* \star *} P<0.001$. 
A

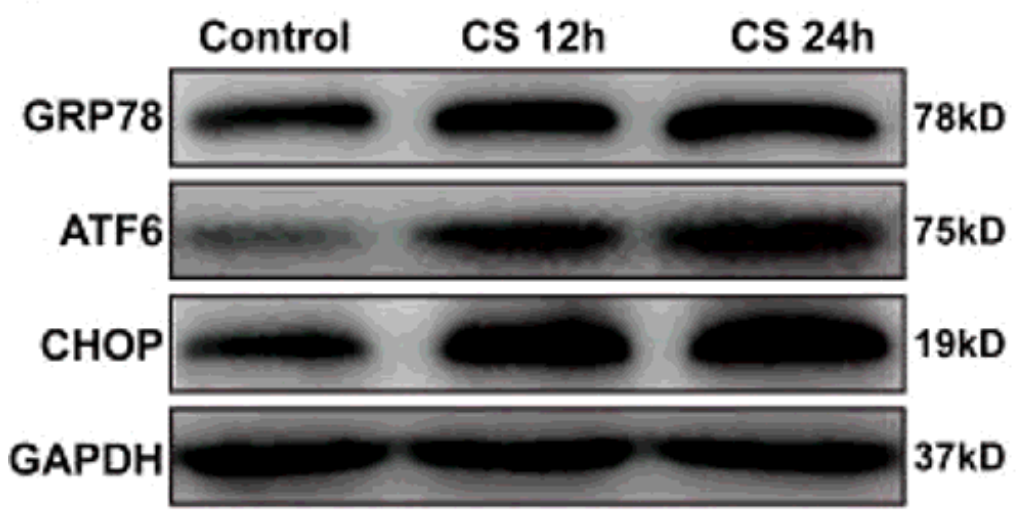

B

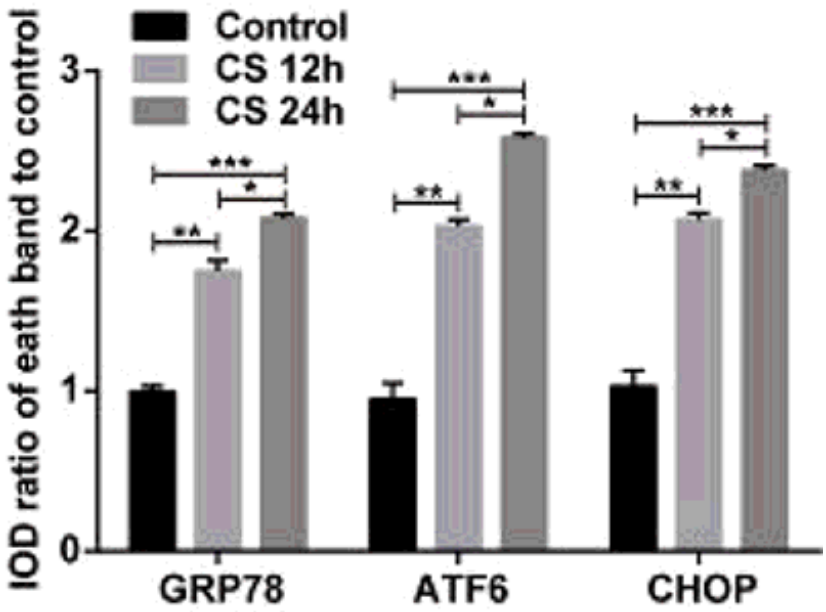

Figure 3

Cold storage-induced endoplasmic reticulum (ER) stress. Expression of glucose-regulated protein 78 (GRP78), activating transcription factor 6 (ATF6), and C/EBP homologous protein (CHOP) in rat hepatic tissues after cold storage for specific time duration. Samples were characterized by Western blot. Images are true representation of the samples. Data are expressed as means + SD of individual groups $(n=6$ rats/group at each time point). ${ }^{*} \mathrm{P}<0.05,{ }^{*} \mathrm{P}<0.01$, ${ }^{* *} \mathrm{P}<0.001$.

A

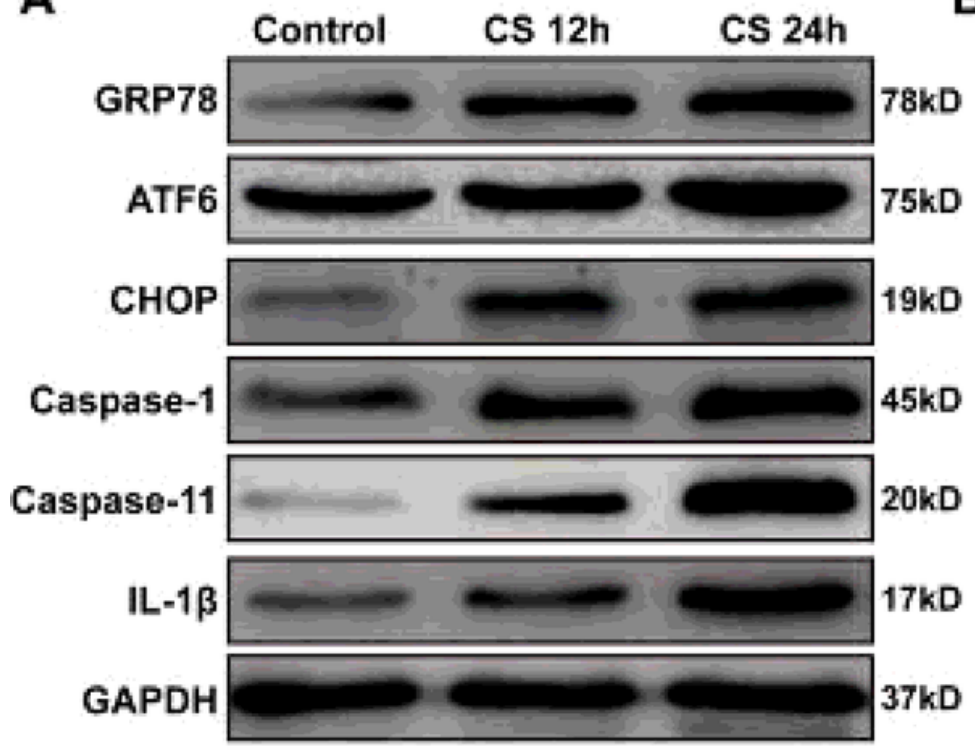

B

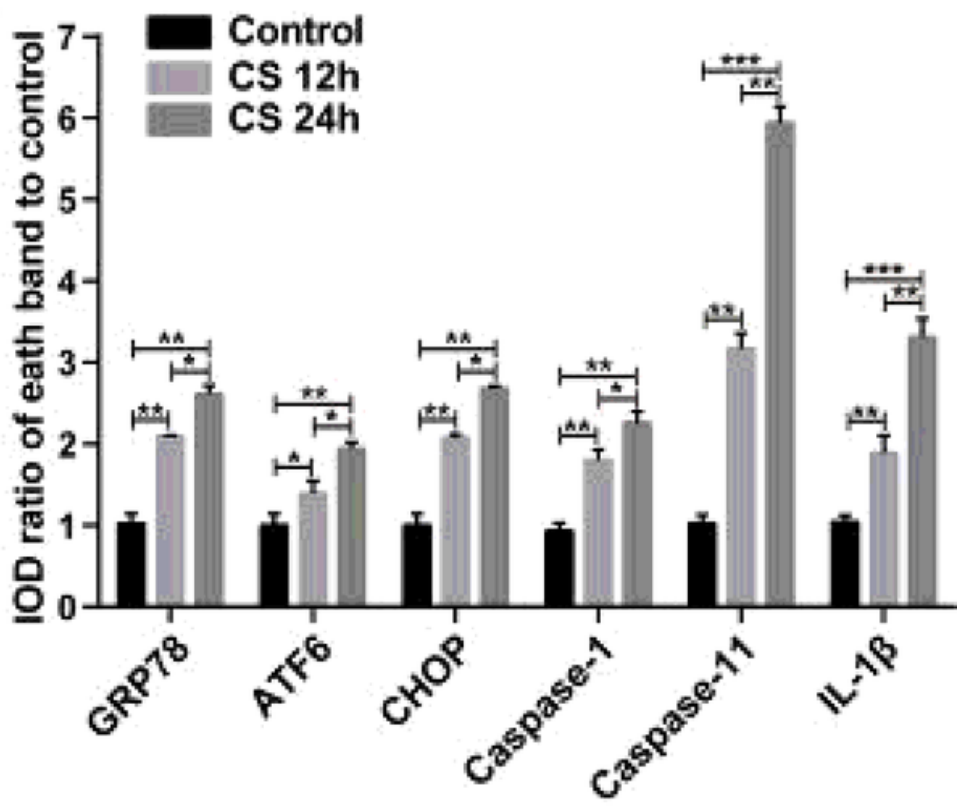

Figure 4

Cold storage-induced ER stress and pyroptosis in BRL cells. BRL cells were cultured under normal conditions as healthy controls or at low temperature $\left(4^{\circ} \mathrm{C}\right)$ to induce injury for the indicated time periods. After treatment, cells were harvested and evaluated by Western blot assay for the levels of GRP78, ATF6, CHOP, caspase-1, caspase-11, and IL-1 1 (A). Images are true representation of the samples. Data are expressed as means $+S D$ of each group of cells from three separate experiments. ${ }^{*} P<0.05,{ }^{\star *} P<0.01$, $\star \star * P<0.001$. 

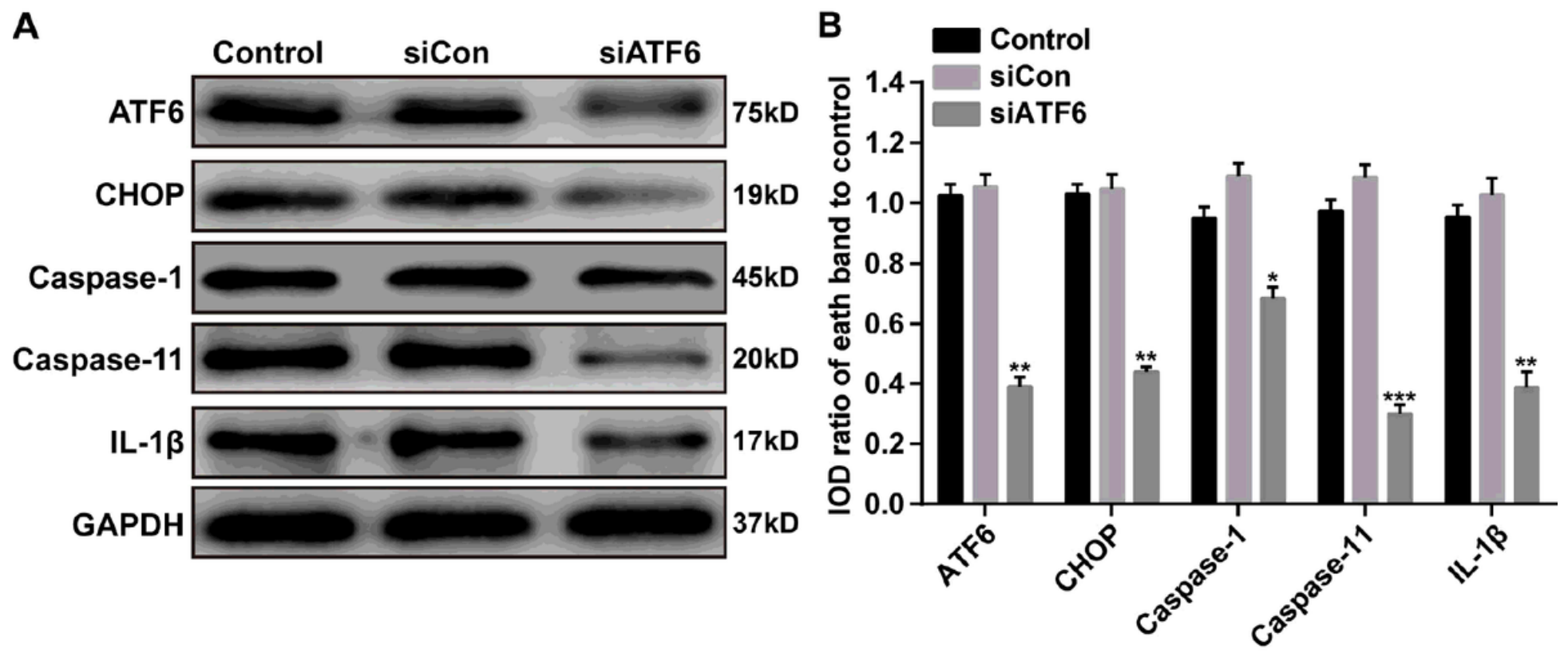

Figure 5

ATF6 siRNA reduces cells' cold-induced injury. Pretreatment with ATF6 siRNA mitigated cells' cold-

induced injury by reducing ATF6 and its downstream effectors CHOP, caspase-1, caspase-11, and IL-1 $\beta$ in BRL. Groups of BRL cells were treated with ATF6 siRNA or control, and subjected to cold storage for $24 \mathrm{~h}$. Levels of ATF6, CHOP, caspase- 1 , caspase-11, and IL-1 $\beta$ were assessed by Western blot assays. ${ }^{*} \mathrm{P}<0.05$, $\star \star P<0.01, * \star * P<0.001$. 